Monatsschr Kinderheilkd 2014 [Suppl 2]

162:218-227

DOI 10.1007/ s00112-014-3206-6

(c) Springer-Verlag Berlin Heidelberg 2014

\title{
Abstracts der 52. Herbsttagung der Deutschen Gesellschaft für Kinderchirurgie (DGKCH)
}

\section{Wissenschaftliche Leitung}

Prof. Dr. med. Guido Fitze, Dresden

\section{Vorträge}

\author{
Hydrozephalus
}

\section{DGKCH-SY-HY-2 \\ Peri- und postnataler Verlauf sowie Therapie des posthämorrhagi- schen Hydrozephalus (PHH) \\ Tröbs R.-B.', Sander V. ${ }^{\text {? }}$ \\ ${ }^{1}$ Marienhospital Herne, Klinikum der Ruhr-Universität Bochum, Klinik für Kinderchirurgie, Herne}

Fragestellung. Ziel der vorliegenden Untersuchung war es, die Besonderheiten des Peri-und postnatalen Verlaufes betroffener Säuglinge, die Dynamik des Geschehens und damit verbundene chirurgische Konsequenzen aufzuzeigen.

Material und Methode. Retrospektiv ermittelten wir 25 zwischen 2007 und 2013 wegen eines $\mathrm{PHH}$ in unserer Klinik primär operierte Säuglinge. Erfasst wurden biometrische Daten, perinatale Ereignisse, die durchgeführte Operation sowie das Kurzzeit-Outcome.

Ergebnisse. Das mediane Geburtsgewicht betrug 980 g (470-3480) und die Schwangerschaftsdauer betrug 27 Wochen (23-39). In 100\% war eine Sectio erfolgt und die Hälfte der Kinder entstammten Mehrlingsgeburten. In 17 Fällen kam es zu schweren peri- und postnatalen Ereignissen. Es dominierten Hirnblutungen der Grad III und IV. Bei 16 Kindern stellten wir die Indikation zur Anlage eines Reservoirs (Liegedauer 17 bis 90 Tage, Komplikation 1/16) und bei 9 wurde primär ein ventrikuloperitonealer Shunt (VPS) installiert. Das mediane Alter in der Gruppe Reservoir betrug 28 vs. 128 Tage in der VPS-Gruppe.

Diskussion. Die Studie illustriert die Bedeutung der kindlichen Unreife in Verbindung mit gravierenden und lebensbedrohlichen peri- und postnatalen Ereignissen für die Entstehung eines PHH. Wir konnten zwei Gruppen mit unterschiedlicher Dynamik des Hydrozephalus identifizieren. Die Langzeitmorbidität für die der Betroffenen ist erheblich.

Schlussfolgerung. Die Primärversorgung mit einem Liquorreservoir ist eine komplikationsarme Möglichkeit der Initialtherapie des PHH. Die Konversionsrate zum Shunt ist jedoch hoch. Bei subakutem Verlauf der Hydrozephalie kann primär ein VPS platziert werden.

\section{DGKCH-SY-HY-4 \\ Zur Verringerung der Infektionsrate nach externer Liquordrainage bei Hydrozephalus-Kindern - was kann man tun?}

Zerche A. ', Großer K. ${ }^{1}$

${ }^{1}$ Klinik für Kinderchirurgie und Kinderurologie, HELIOS Klinikum Erfurt, Erfurt, Deutschland

Hintergrund. Die externe Liquordrainage ist eine therapeutische Option bei Kindern mit posthämorrhagischem Hydrozephalus, aber auch bei anderen akuten Hydrozephaluserkrankungen. Infektionen sind dabei im Verlauf eine häufige Komplikation. Explizite Guidelines bezüglich des operativen Procedere können helfen, die Infektionsrate zu verringern.

Material und Methode. Wir überschauen retrospektiv 59 Kinder, die mit einer EVD behandelt wurden. Der Beobachtungszeitraum begann 2001 und endete 2012. Es wurden 18 Frühgeborene, 14 terminlich Geborene bis zum Alter von einem Jahr und 27 Kinder zwischen 1 und 15 Jahren ausgewertet. Die Liegedauer der EVD variierte zwischen 8 und 146 Tagen, mittlere Liegedauer 20 Tage. In 3 Fällen bedurfte es besonders langer EVD- Drainagedauer einmal wegen Ösophagusatresie, einmal wegen Gastroschisis und einmal wegen schwerer NEC mit intraabdominaler Perforation und Peritonitis. Wir nutzten einen lang getunnelten EVD-Kanal, beginnend vom Bohrloch bis zum oberen Rumpf. Die Bakterienaszension zum Ventrikel wird mit dieser Methode durch eine lange Tunnelungsstrecke reduziert. Es wurde an der Austrittsstelle eine sehr kleine Inzision vorgenommen und eine beidseitige Katheterfixation mit monofilem Prolenfaden $4 \times 0$, um Katheterbewegungen an der Hautaustrittstelle zu verhindern. Damit wird die Bildung eines Biofilms am Katheter und im Hauttunnel um den Katheter herum reduziert. Auf kontinuierlich trockene Wundverhältnisse ohne Weichteilirritation und zusätzlicher Verbandsicherung der Katheterlage ist zu achten.

Ergebnisse. Wir beobachteten im Untersuchungszeitraum 4 Fälle mit Infektionen (7\%). Drei dieser Kinder waren Frühgeborene mit hoher Immuninkompetenz, ein Kind war älter als ein Jahr alt. Die Infektionsrate unter den Frühgeborenen und Säuglingen lag bei $9 \%$. In allen Fällen entfernten wir umgehend das System und es wurde dreimal eine Neuanlage an anderer Stelle der Hirnventrikel durchgeführt. Alle Infektionen konnten mit Vancomycingaben beherrscht werden. Die Gesamtletalität unter den 59 Kindern lag bei $2 \%$ (1/59 Kindern).

Schlussfolgerung. Sorgfältige asetptische Operation, lange Tunnelungsstrecke und optimale Wundpflege zusammen mit antibiotischer Prophylaxe können das Infektionsrisiko bei EVD verringern. 


\section{Bauchwanddefekte}

\section{DGKCH-SY-BD-4}

Erfurter Erfahrung mit der terminierten Geburt bei Laparoschisis 2011 bis 2014

Winterhalter J.', Nöth N.', Cremer M.', Großer K. ${ }^{1}$

'Klinik für Kinderchirurgie und Kinderurologie, HELIOS Klinikum Erfurt, Erfurt, Deutschland

Einleitung. Die Versorgung einer Laparoschisis stellt weiterhin eine kinderchirurgische Herausforderung dar. Die Option der terminierten Geburt wird zunehmend angewandt.

Zielstellung. Darstellung der Erfahrungen mit der terminierten Geburt bei Laparoschisis von 2011 bis 2014 in der Kinderchirurgie Erfurt.

Methode. Beschreibung der postoperativen Verläufe von 6 Patienten nach terminierter Geburt in der 34. bis 35. SSW. Patientenkollektiv: 5 Jungen und 1 Mädchen. Geburtsgewicht: von 1970-3120 g. Als Zusatzdiagnosen traten auf: einmal Jejunalatresie, einmal Vanishing-Bowl.

Ergebnisse. Fünf Kinder zeigen gutes Gedeihen und reizlose Narben. Zwei Kinder wurden wegen Ileus nachoperiert. Bei einem Kind steht aktuell noch die Versorgung einer sekundären Umbilicalhernie aus. Ein Kind ist an einer Hickman-Katheter-Sepsis extra muros verstorben. Postnatale Komplikationen: 1-mal Amnioninfektionssyndrom, 1-mal passagere prärenale akute Niereninsuffizienz.

Diskussion. Mit der zeitnahen definitiven Versorgung wird das Infektionsrisiko minimiert. Der Kostaubau kann frühzeitig gestartet werden. Es sind keine geplanten enteralen Zweiteingriffe nötig. Die kalkulierte Frühgeburtlichkeit hat den Verlauf nicht negativ beeinflusst. Allerdings zeigt sich im Verlauf zu 50\% eine Umbilicalhernie, die leicht versorgt werden kann.

Schlussfolgerung. Die terminierte Geburt und der primäre Bauchdeckenverschluss können hinsichtlich der in unserer Klinik gemachten Erfahrungen als Behandlungsmethode empfohlen werden.

\section{DGKCH-SY-BD-6}

\section{Gastroschisis im Silo - enteral ernähren oder nicht?}

\section{Lawrenz K. ', Thiel K. ${ }^{2}$}

${ }^{1}$ Helios Klinikum Krefeld, Klinik für Kinderchirurgie, Krefeld, ${ }^{2}$ Helios Klinikum Krefeld, Kinderzentrum, Krefeld

In dem internationalen kinderchirurgischen Forum „Pedsurg@yahoogroups.com“" wurde kürzlich die Frage erörtert, ob Neugeborene mit einer mit einem Federsilo versorgten Gastroschisis gefüttert werden sollen oder nicht. Die Mehrzahl der Diskussionsteilnehmer lehnte dies ab. Klinische Studien zu diesem Thema gibt es nicht. Die grundsätzlichen Vorteile der enteralen gegenüber einer parenteralen Ernährung sind aber hinlänglich bekannt. Unsere positive Erfahrung mit dem unmittelbar postpartalen Nahrungsaufbau mit Muttermilch ad libitum wird anhand einer Kasuistik dargestellt und unter Berücksichtigung der in aktuellen Publikationen auffindbaren Daten reflektiert.

\section{Freie Vorträge}

\author{
DGKCH-FV-KC-1 \\ Relapsing episodes of fever, exanthema, arthralgias and lymphade- \\ nopathy in a boy with severe course of pathological susceptibility \\ to infection - from autoinflammatory disease (AID) to primary \\ immunodeficiency (PID)
}

\section{Dueckers G. ${ }^{1}$, Kramm C. ${ }^{2}$, Kühnle I. ${ }^{2}$, Kuemmerle-Deschner J. ${ }^{3}$, Niehues T. ${ }^{1}$ ${ }^{1}$ HKK HELIOS Klinikum Krefeld, Krefeld, ${ }^{2}$ Universitätsklinikum, Göttingen, ${ }^{3}$ Universitätsklinikum, Tübingen}

Case report. A 3-year-old male of a non-consanguineous family presented with recurrent episodes of fever, exanthema and arthralgia and positive ANA titer. Subsequently the boy was admitted to hospital because of a pulmonary abscess and mediastinal lymphadenopathy (histopathology: chronic granulomatous inflammation). As no pathogen could be isolated in lung biopsy, the patient has been suspected for AID. A heterozygous mutation (Q703K) in $\mathrm{NLRP}_{3}$ supported AID and therefore prednisolone and anakinra were administered. Not until the commencement of canakinumab, fever, exanthema and arthralgia disappeared. Pathological susceptibility to infection was diagnosed after the occurrence of following complications within the following six months 1) mycoplasma related peribronchitis, 2) complicated cervical lymphadenopathy with abscess formation (isolated pathogen: Neisseria mucosa), postoperative disturbance of wound healing and formation of fistulae, 3) re-occurrence of fever, 4) persistent, painful, severe mesenteric lymphadenopathy with lymph node abscess, 5) severe pyogenic appendicitis (histopathology: chronic granulomatous inflammation), 6) diarrhea and 7) inguinal lymph node abscess (isolated pathogen: Serratia marescens). At that time Canakinumab was withdrawn. Immunological investigation (oxidative burst test) revealed the diagnosis of chronic granulomatous disease, a primary immunodeficiency. Beside pathogen targeted treatment, prophylactic anti-infectious therapy (itraconazole and trimethoprim) was initiated and the patient was referred to a bone marrow transplantation unit.

Conclusion. Primary immunodeficiencies might present with autoinflammatory symptoms and are careful evaluation of differential diagnosis is mandatory. Pathological susceptibility to infection in patients with AID, should lead to immunological investigation immediately. Nevertheless, the value of anti-IL1 inhibition in chronic granulomatous disease should be critically investigated, as IL1 receptor blockade might reduce inflammation in CGD.

\section{DGKCH-FV-KC-2}

Current best practice options in the management of hypospadias of the Société Internationale d'Urologie (SIU) 2014

\section{Springer $A .^{\prime}$}

${ }^{1}$ Klinische Abteilung für Kinderchirurgie, Wien, Österreich

Hypospadias is one of the most common malformations of the penis. Depending on the severity of the defect there can be cosmetic, functional and psychological long-term effects for the patient. A cooperative working group consisting of pediatric urologists with an interest in hypospadias gathered under the auspices of the Société Internationale d'Urologie (SIU) in an effort to produce the update of current best practice options for the treatment of hypospadias based on a systematic review using MEDLINE between 1990 and 2013. Whenever possible, statements have been classified in terms of level of evidence and grade of recommendation as proposed by the Centre for Evidence-Based Medicine. However, due to the limited availability of randomized controlled trials and high quality studies this document will largely be a consensus document including expert opinion. These guidelines include indications for surgery, classification, documentation, diagnostic tests, the use of preoperative androgens, preferred techniques for the correction of 
mild and severe hyposopadias, urinary drainage and wound dressing, the use of perioperative antibiotics, and finally follow-up and assessment of outcomes. The 2014 SIU current best practice options in the future could be assistance for clinicians in the treatment of hypospadias and towards standardization of treatment.

\section{DGKCH-FV-KC-3}

Funktionelle Störungen der Halswirbelsäule als Hauptursache für den lagebedingten Plagiocephalus

\section{Küsgen B. ${ }^{1}$, Bahr M. ${ }^{2}$, Biedermann H. ${ }^{1}$}

${ }^{1}$ Privatpraxis Huhnsgasse, Orthopädie/Manualmedizin, Köln, ${ }^{2}$ Marienhospital Herne, Klinikum der Ruhr-Universität Bochum, Kinderchirurgie, Herne

Hintergrund. Die zugrundeliegenden Ursachen für den Plagiocephalus werden weiterhin kontrovers diskutiert. Meist liegen diese in einer reversiblen Funktionsstörung (KISS) der sog. Kopfgelenke (Okziput/C1 bzw. C1/C2). Die frühzeitige Diagnosestellung und Behandlung macht hinsichtlich möglicher Spätfolgen Sinn. Die Autoren verfolgen einen manualmedizinischen Ansatz zur Behandlung.

Ziel der Pilotstudie. Aussagen zur Effektivität dieser Behandlungsmethode zu machen und Informationen zu Ursachen zu generieren.

Methoden. 72 Babies (Alter 3-6 Monate) wurden in die Studie eingeschlossen. 6-Wochen-, 3- bzw. 6-Monats-Follow-up-Daten wurden erhoben. Die Babies wurden entsprechend einem standardisierten Studienprotokoll gesehen: anamnestische Befragung der Eltern, manualmedizinische Untersuchung, a.p.-Röntgenbild der HWS, Behandlung mittels .anueller Techniken. 54\% Jungen, 46\% Mädchen wurden untersucht, mittleres Alter: 5,1 Monate.

Ergebnisse. Sechs Wochen/3 bzw. 6 Monate nach Behandlung wurde die Besserung der Hauptbeschwerden in einer 4-Stufen-Skala abgefragt, die in $88 \%$ der Fälle nach 6 Wochen eine gute/exzellente Verbesserung ergab, was im 3-Monats-/6-Monats-Follow-up stabil blieb und sich in keinem der Fälle verschlechterte. Die Auswertung der anamnestischen Daten ergab, dass komplikationsreiche Geburtsvorgänge mögliche Ursache für die Entwicklung von KISS sind: Nur in 12\% fand eine unkomplizierte vaginale Entbindung statt, $78 \%$ waren Sectiones bzw. vaginale Operationen. In $47 \%$ wurde „kristellert“ (Druck von außen auf den mütterlichen Bauch). Nur 22\% der Babies waren unbehandelt, $51 \%$ vorher osteopathisch behandelt.

Schlussfolgerung. Die manualmedizinische Behandlung der HWS von plagiocephalen Babies ist eine effektive Methode, um eine frühzeitige, nachhaltige Verbesserung sowohl der Kopfform als auch der HWSFunktion zu erreichen.

\section{DGKCH-FV-KC-4 \\ Der Chondroepitrochlearis-Muskel - ein Atavismus mit kinder- chirurgischer Relevanz}

Tröbs R.-B. ${ }^{1}$

'Marienhospital Herne, Klinikum der Ruhr-Universität Bochum, Kinderchirurgische Klinik, Herne

Fragestellung. Wir berichten über eine extrem seltene Varietät des Pectoralis-Muskels.

Material und Methode. Fallbericht.

Ergebnisse. Der 7 Monate alte Knabe wurde mit eine Strangbildung über der rechten Axilla vorgestellt, der sich bei Abduktion und Elevation des linken Armes scharf abzeichnete. Dieser ging vom oberen Anteil des M. pectoralis aus, überkreuzte die Axilla und inserierte am Epicondylus ulnaris humeri. Wir führten die Resektion dieses strangförmigen Muskels aus. Komplikationen traten nicht ein. Das Resektat bestand zu einem Drittel aus einem schmächtigen, spindelförmigen Skelett-Muskel, der sich in eine lange, ca. zwei Drittel der Gesamtlänge ausmachende Sehne fortsetzte.
Diskussion. Der M. chondroepitrochlearis ist wahrscheinlich ein entwicklungsgeschichtliches Residuum. So könnte es sich um ein stammesgeschichtliches Äquivalent zum Extensor plicae alaris der Vögel handeln. Schlussfolgerung. Die Indikation zur Operation leitet sich aus einer funktionellen Einschränkung, möglichen neurologischen Problemen sowie der ungewohnten Kosmetik ab.

\section{DGKCH-FV-KC-5}

Postoperatives Outcome nach Korrektur der Ösophagusatresie in Deutschland: Analyse von Krankenkassendaten und internationaler Vergleich

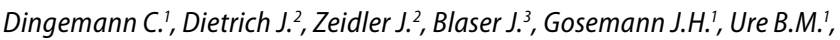
Lacher M. ${ }^{1}$

'Zentrum Kinderchirurgie Hannover, Medizinische Hochschule Hannover, Hannover, ${ }^{2}$ Center for Health Economics Research Hannover (CHERH), Institut für Versicherungsbetriebslehre, Leibniz Universität Hannover, Hannover, ${ }^{3}$ Techniker Krankenkasse, Landesvertretung Niedersachsen, Hannover

Fragestellung. Die Behandlung von Patienten mit Ösophagusatresie (ÖA) ist in Deutschland nicht zentralisiert. Hypothese war, dass die multi-institutionale Behandlung im internationalen Vergleich zu schlechteren postoperativen Ergebnissen führt.

Material und Methoden. Anonymisierte Analyse von Krankenkassendaten (Techniker Krankenkasse). Alle ÖA-Patienten von 01/2007 bis 08/2012 wurden eingeschlossen. Die behandelnden Kliniken wurden anhand universitärer Anbindung, Anzahl der Oberärzte, Klinikbetten und Frühgeborener $\leq 1500$ g klassifiziert. Operative Daten, Patientencharakteristika, Reoperationen innerhalb eines Jahres nach Ersteingriff und Komplikationen wurden erfasst. Die postoperativen Resultate wurden mit der aktuellen Literatur verglichen.

Ergebnisse. 75 ÖA-Patienten wurden in 37 Kliniken behandelt. Anastomoseninsuffizienzen traten bei 2 Patienten (3\%) auf, was mit jüngsten Publikationen vergleichbar ist (2-8\%). Fistelrezidive wurden bei 5 Patienten ( $7 \%$ ) dokumentiert, was ebenso mit Literaturdaten übereinstimmt (2-10\%). Anastomosenstrikturen traten bei 43 Patienten $(57 \%)$ und damit im internationalen Vergleich (22-78\%) häufig auf. Die Inzidenz postoperativer Komplikationen korrelierte nicht mit den strukturellen Merkmalen der Kliniken ( $\mathrm{p} \geq 0,05)$.

Diskussion. Anhand der Datenbank einer Krankenkasse konnten objektive postoperative Ergebnisse nach ÖA-Korrektur analysiert werden. Trotz multi-institutionaler Behandlung waren die Komplikationsraten vergleichbar mit denen großer internationaler Zentren. Es bestand keine Korrelation zwischen Komplikationsrate und strukturellen Merkmalen der behandelnden Kliniken. Die fehlende Zentralisierung in Deutschland geht somit im Vergleich zum Outcome internationaler Zentren nicht mit ungünstigeren postoperativen Ergebnissen einher.

\section{DGKCH-FV-KC-6}

Pulmonologische Langzeitfolgen und ihr Zusammenhang mit Frühkomplikationen bei Patienten mit korrigierter Ösophagusatresie

Dittrich R. ', Stock P. ${ }^{2}$, Rothe K.', Degenhardt P. ${ }^{1}$

${ }^{1}$ Charité Universitätsmedizin Berlin, Klinik für Kinderchirurgie, Berlin, ${ }^{2}$ Charité Universitätsmedizin Berlin, Klinik für Pädiatrie m. S. Pneumologie und Immunologie, Berlin

Fragestellung. Ziel dieser prospektiven Studie war die Analyse und Evaluation pulmonologischer Langzeitfolgen und ihr Zusammenhang mit Frühkomplikationen bei Patienten mit korrigierter Ösophagusatresie. Material und Methoden. Bei 27 Patienten mit einer korrigierten Ösophagusatresie im Alter von 5 bis 20 Jahren wurden Lungenfunktionsuntersuchungen, sowie Laufbandbelastungstests durchgeführt. Daten zum operativen und perioperativen Ergebnis, zu pulmonologischen und gastroösophagealen Komplikationen, sowie zur körperlichen Entwicklung wurden anhand von Fragebögen, sowie durch Einsicht der Patientenakten erfasst. 
Ergebnisse. 63\% der Studienteilnehmer zeigten eine insgesamt abnorme Lungenfunktion. Restriktive Ventilationsstörungen (rein restriktiv oder kombiniert) wurden bei $41 \%$ der Teilnehmer, obstruktive Ventilationsstörungen (rein obstruktiv oder kombiniert) bei $48 \%$ gemessen. Mit einem Z-Score von 1,40 (Mittelwert) für die Laufbandbelastung war die körperliche Belastbarkeit signifikant kleiner als der Durchschnitt der Referenzwertpopulation. Signifikante Korrelationen bestanden zwischen dem Vorliegen von restriktiven Ventilationsstörungen und der Distanz der Ösophagusblindsäcke, der Dauer postoperativer Beatmung, einem gastroösophagealen Reflux, sowie rezidivierende Aspirationspneumonien im Kleinkindalter.

Schlussfolgerung. Eine Ösophagusatresie und ihre (Früh-)Komplikationen haben signifikante Auswirkungen auf das pulmonologische Langzeitergebnis. Eine langfristige und regelmäßige Nachsorge von Patienten mit korrigierter Ösophagusatresie ist von besonderer Bedeutung, um das Entstehen und Fortschreiten assoziierter Pathologien (Ventilationsstörungen, gastroösophagealer Reflux u. a.) frühzeitig zu erkennen und durch geeignete Maßnahmen vorbeugen zu können.

\section{DGKCH-FV-KC-7}

Akute Appendizitis bei Kindern und Jugendlichen in Deutschland: Analyse einer Krankenversicherungsdatenbank

Gosemann J.-H.', Lange A. ', Zeidler J. ${ }^{2}$, Blaser J. ${ }^{3}$, Dingemann C. ', Ure B.M.', Lacher $M{ }^{1}$

'Zentrum Kinderchirurgie Hannover, Medizinische Hochschule Hannover, Hannover, ${ }^{2}$ Leibniz Universität Hannover, Center for Health Economics Research Hannover (CHERH), Institut für Versicherungsbetriebslehre, Hannover, ${ }^{3}$ Techniker Krankenkasse, Landesvertretung Niedersachsen, Hannover

Fragestellung. Die akute Appendizitis behandeln in Deutschland Kinderchirurgen $(\mathrm{KCH})$ und Allgemeinchirurgen $(\mathrm{ACH})$. Wir analysierten die Behandlungsmodalitäten und Ergebnisse beider chirurgischer Disziplinen.

Material und Methode. Anonymisierte Analyse von Krankenkassendaten (Techniker Krankenkasse, 10\% der Bevölkerung) aller Patienten (Alter 4-17 Jahre) mit dem OPS code „Appendektomie“ aus dem Zeitraum 2010-2012. Eine log. Regressionsanalyse erfolgte für das „Risiko einer chirurgischen Komplikation" (Variablen: KCH/ACH, Alter, Geschlecht, Perforation, Technik, Komplikationen).

Ergebnisse. Daten von 8110 Patienten [KCH: $n=1937$ (24\%); ACH: $n=6173$ (76\%)] wurden erfasst. $\mathrm{KCH}$ versus $\mathrm{ACH}$ behandelten häufiger jüngere Patienten (4-13 Jahre; 77\% vs. 41\%) und seltener Adoleszente (14-17 Jahre; $23 \%$ vs. $59 \%)(\mathrm{p}<0,001)$. ACH operierten häufiger laparoskopisch (79\% vs. $61 \%$; $\mathrm{p}<0,001)$ mit einer signifikant niedrigeren Konversionsrate ( $1 \%$ vs. $3 \% ; \mathrm{p}<0,001)$. Nach kinderchirurgischen Operationen war die Komplikationsrate während des Erstaufenthaltes und die Wiederaufnahmerate signifikant höher (2,9\% vs. $1,7 \%$ und $3,4 \%$ vs. $2,2 \%$; $p<0,01)$. Die log. Regressionsanalyse ergab keine Unterschiede zwischen den Disziplinen bezüglich des Risikos einer Komplikation bei jüngeren Patienten (4-11 Jahre). Das Risiko für eine Wiederaufnahme aufgrund einer chirurgischen Komplikation war für adoleszente Patienten der KCH signifikant höher

Schlussfolgerung. Die Ergebnisqualität der Behandlung der akuten Appendizitis im Kindes- und Jugendalter ist in Deutschland sehr gut. Für jüngere Patienten gilt dies unabhängig von der behandelnden chirurgischen Disziplin. Für adoleszente Patienten bestand in dieser Studie bei der Behandlung durch $\mathrm{KCH}$ ein höheres Risiko für eine Wiederaufnahme wegen einer chirurgischen Komplikation.

\section{DGKCH-FV-KC-8 \\ Chirurgische Aspekte der Behandlung von GvHD des Darmes im Kindesalter}

Schneider A. ', Kühl J.-S. ${ }^{2}$, Rothe K. ${ }^{1}$

${ }^{1}$ Klinik für Kinderchirurgie, Charité, Universitätsmedizin Berlin, Berlin, ${ }^{2}$ Klinik für Pädiatrie m. S. Onkologie und Hämatologie, Charité, Berlin

Hintergrund. Die Behandlung einer höhergradigen Graft-versus-HostDisease (GvHD) des Darmes bei Kindern nach Stammzelltransplantation stellt eine Herausforderung der pädiatrischen Onkologie dar, die chirurgische Therapie i. S. der Resektion betroffener Abschnitte gilt als Ultima ratio.

Methoden. Diesbezüglich haben wir unsere Patienten der letzten 8 Jahre betrachtet und 7 Kinder (3 Mädchen/4 Jungen) identifiziert, die aufgrund einer GvHD chirurgisch therapiert wurden, das mittlere Alter zum OP-Zeitpunkt betrug 11,9 Jahre. Im Folgenden sollen die diagnostischen Möglichkeiten und Aussagen präoperativ, die Problematik der Indikationsstellung sowie des Zeitpunkts der Operation, die Komplikationen und notwendigen Re-Eingriffe und schließlich das Outcome der Kinder bis hin zur Kurzdarm-Situation analysiert und vorgestellt werden.

Schlussfolgerung. Sichere, evidenzbasierende Handlungsempfehlungen lassen sich aufgrund der kleinen, heterogen und durch die Grunderkrankung dominierten Patientengruppe nicht ableiten. Wir denken, dass eine chirurgische Therapie als Ultima-ratio-Maßnahme weiterhin sinnvoll und durchführbar erscheint, dass der Wahl des Operationszeitpunkts besondere Bedeutung zukommt und dass dann die radikale Entfernung aller betroffener Abschnitte die einzige Option darstellt.

\section{DGKCH-FV-KC-9}

\section{Fournier-Gangrän im Kindesalter}

Jafarzade G. ${ }^{1}$, Varol E. ${ }^{2}$, Müller C. ${ }^{2}$, Bahlmann H. ${ }^{2}$, Barthlen W. ${ }^{2}$

${ }^{1}$ Universitätsmedizin Greifswald, Kinderchirurgie, Greifswald, ${ }^{2}$ Universitätsmedizin Greifswald, Greifswald

Einleitung. Die Fournier-Gangrän ist eine nekrotisierende Fasciitis des Perineums durch eine Mischinfektion aus aeroben und anaeroben Erregern. Im Erwachsenenalter beträgt die Letalität bis zu $34 \%$. Im Kindesalter tritt sie selten auf.

Fallbericht. Bei einem 14-jährigen Mädchen wurde bei anhaltenden Schmerzen im Steißbeinbereich auswärts die Exzision eines Pilonidalsinus durchgeführt. Die Wunde wurde offen behandelt. Bei postoperativ anhaltenden Schmerzen und Rötung im Wundbereich wurden Antibiotika und Analgetika verabreicht, darunter auch Metamizol. Vier Wochen nach dem Eingriff wurde das Kind notfallmäßig in die Kinderchirurgie der Universitätsmedizin Greifswald verlegt. Bei Aufnahme zeigte sich das Vollbild des septischen Schocks mit hohen Temperaturen, Apathie, nicht messbarem Blutdruck, Anurie und respiratorischer Insuffizienz. Zudem bestand eine vollständige Agranulozytose. Lokal bot sie das Bild der ausgeprägten Fournier-Gangrän mit tiefreichenden Nekrosen bis in den Unterbauch und in beide Oberschenkel auslaufend. Urethra, Vagina und Rektum waren intakt. Nach adäquater Schocktherapie erfolgte die radikale Nekrektomie und Vakuumtherapie. Die Agranulozytose, die sich als allergische Reaktion auf die langdauernde Metamizolgabe herausstellte, wurde mit Granulozytentransfusionen behandelt. Im Laufe der folgenden Wochen erfolgten multiple Nekrektomien, Verbandwechsel, Verschiebelappenplastiken und Spalthauttransplantationen zur Deckung der ausgedehnten Wundflächen. Das Mädchen konnte in restitutio ad integrum nach Hause entlassen werden.

Schlussfolgerung. Auch heute noch stellen bei Kindern die FournierGangrän sowie die Metamizol-induzierte Agranulozytose lebensgefährliche Komplikationen dar. 
Poster

\section{Posterbegehung 1}

\section{DGKCH-PO-KC-1 \\ Die akute Appendizitis im Kindesalter - eine sonographische Blick- diagnose}

\section{Eckert $K^{\prime}$}

'Elisabeth-Krankenhaus Essen, Klinik für Kinderchirurgie, Essen

Einleitung. Die akute Appendizitis (AA) des Kindes ist auch für den erfahrenen Untersucher nicht selten eine diagnostische Herausforderung. Auch laborchemische Entzündungsparameter sind nur von geringer Aussagekraft. Zusammen mit einer adäquaten Anamnese und klinischen Untersuchung erreicht der Ultraschall (US) eine hohe Sensitivität und Spezifität, die eine frühe Diagnosestellung ermöglicht, aber auch unnötige Operationen vermeiden hilft. Grundkenntnisse des Appendix-US sind somit für jeden Kinderchirurgen unerlässlich. In diesem Beitrag sollen die US-Charakteristika sowohl der unauffälligen als auch der entzündeten Appendix im Detail dargestellt werden.

Material und Methoden. Nach orientierender US-Beurteilung des Gesamtabdomens mit einer Sektorsonde erfolgt der gezielte Appendix-US mit einer Linearsonde. Bei typischer Lage der Appendix kann diese zumeist ventral des M. psoas gefunden werden. Aber auch an eine retrozökale oder atypische Lage der Appendix ist zu denken. Die sonographische Darstellung klinisch wichtiger Differenzialdiagnosen belegt den zusätzlichen Wert einer adäquaten Abdomen-Sonographie.

Ergebnisse. Sensitive US-Parameter die für eine AA sprechen sind das sog. Umgebungsinfiltrat sowie eine Auflockerung bis Aufhebung der Wandschichtung der Appendix. Komprimierbarkeit, Perfusionsmessungen aber auch quantitative Messwerte (z. B. Durchmesser) sind von untergeordneter Bedeutung.

Schlussfolgerung. Mit Kenntnis der normalen und auch der pathologischen Appendixmorphologie gelingt eine gute Beurteilung der Appendix und ermöglicht eine zeitgerechte Therapie bei AA. Eine adäquate abdomensonographische Expertise auch des Kinderchirurgen hat somit einen hohen Stellenwert, die deshalb in der Aus- und Weiterbildung auch entsprechend berücksichtigt werden muss.

\section{DGKCH-PO-KC-2}

Appendizitis im Säuglingsalter - eine diagnostische Herausforderung

\section{Amasheh K.', Vogt H. ', Tröbs R.-B.' \\ 'Klinik für Kinderchirurgie RUB, Herne}

Hintergrund. Die akute Appendizitis ist eine der typischen Erkrankungen im Kindes- und Jugendalter. Jedoch treten nur circa $4 \%$ vor dem 3. und weniger als $1 \%$ vor dem 2. Lebensjahr (Puri et al., J. Ped. Surg 1978;13:173-174) auf. Aufgrund der geringen Inzidenz in dieser Altersgruppe und deren Besonderheiten, liegen bei Diagnosestellung meist eine Perforation oder ein perityphlitischer Abszess vor.

Material und Methoden. Retrospektive Evaluation unserer Patienten, die in den letzten beiden Jahren mit einer akuten Appendizitis behandelt wurden ( $\mathrm{n}=216)$. Zwei Patienten waren jünger als $1 \mathrm{Jahr}(0,9 \%)$.

Ergebnisse. Patient 1: Ein 6 Monate altes Mädchen, das primär mit der Verdachtsdiagnose einer Gastroenteritis behandelt wurde: Bei weiterer Exazerbation unter konservativer Therapie fiel in der Verlaufssonographie ein perityphlitischer Abszess auf, der bei Erstvorstellung 48 Stunden zuvor noch nicht apparent war (CRP 11,2 mg/dl, Leukos 18,3/ nl). Patient 2: Ein 5 Monate alter Junge, bei dem die Entzündung des Wurmfortsatzes erst bei Entwicklung einer Begleitperitonitis erst als akutes Skrotum aufgrund eines offenen Processus vaginalis peritonei symptomatisch wurde. (CRP 26,5 mg/dl, Leukozyten 21/nl).
Diskussion. Während die Therapie der ersten Patientin aufgrund der Verdachtsdiagnose Gastroenteritis - der wohl häufigsten Differenzialdiagnose der Appendizitis - verzögert wurde, war beim zweiten Patient eine schmerzhafte Hydrocele im Verbund mit Allgemeinsymptomen wegweisend.

Schlussfolgerung. Je jünger der Patient ist umso mehr ist die Appendizitis ein diagnostisches Chamäleon und bedarf äußerster diagnostischer Sorgfalt.

\section{DGKCH-PO-KC-3}

Klinische Manifestation des symptomatischen Meckel-Divertikels im Kindesalter - vier Fallberichte

\author{
Eckert K.', Klompen J.', Radeloff E.', Liedgens P.'
}

'Elisabeth-Krankenhaus Essen, Klinik für Kinderchirurgie, Essen

Einführung. Das Meckel-Divertikel (MD) ist eine angeborene Fehlbildung des Magen-Darm-Trakts, das nur bei etwa $4 \%$ der Betroffenen symptomatisch wird. Das MD manifestiert sich klinisch äußerst variabel und oft unspezifisch, so dass es dadurch anderen, häufigeren Krankheitsbildern ähneln kann. Anhand von vier eigenen Fallberichten sollen mögliche Komplikationen des MDs veranschaulicht werden. Fall 1. Ein 11-jähriger Junge wurde mit akuten, kolikartigen Bauchschmerzen und Erbrechen vorgestellt. Sonographisch zeigte sich eine ileoileale Invagination. Intraoperativ fand sich ein invaginiertes MD im Ileum und wurde durch Keilresektion entfernt.

Fall 2. Ein 10-jähriges Mädchen wurde mit progredienten Bauchschmerzen und zuletzt galligem Erbrechen vorgestellt. Radiologisch/sonographisch zeigte sich das Bild eines mechanischen Ileus. Intraoperativ zeigte sich ein Dünndarmvolvulus durch einen persistenten Ductus omphaloentericus. Nach Detorquierung erfolgte die Resektion des Ductus mit Keilexzision am Ileum.

Fall 3. Ein 10-monatiges Mädchen wurde mit peranaler Blutung vorgestellt. Der Eingangs-Hb lag bei 6,5 g/dL. Eine Tc99-Szintigraphie erhärtete die Verdachtsdiagnose eines $\mathrm{MD}$, das sich intraoperativ als ein breitbasiges, ulzeriertes MD bestätigte und reseziert wurde.

Fall 4. Ein 2,5-jähriges Mädchen wurde mit starken, rechtsseitigen Unterbauchschmerzen vorgestellt, die klinisch, laborchemisch und sonographisch auf eine Appendizitis hindeuteten. Der intraoperative Befund zeigte jedoch ein abszediertes MD, das reseziert wurde.

Schlussfolgerung. Die präoperative Diagnose eines symptomatischen MDs gelingt nur selten. Besonders im Kleinkindalter ist bei unklaren klinischen und bildgebenden Befunden immer auch an ein MD zu denken und bei gegebener Klinik eine OP-Indikation großzügig zu stellen.

\section{DGKCH-PO-KC-4}

Hernia inguinalis mit ungewöhnlicher Ursache - juveniler Granulosazelltumor bei einem 6 Monate alten Mädchen

Weber F.D. ${ }^{1}$, Frühwald M.C. ${ }^{2}$, Märkl B. ${ }^{3}$, Schneider D. ${ }^{4}$, Schuster T. ${ }^{1}$ 'Klinikum Augsburg, Kinderchirurgische Klinik, Augsburg, ${ }^{2}$ Klinikum Augsburg, I. Klinik für Kinder und Jugendliche, Augsburg, ${ }^{3}$ Klinikum Augsburg, Institut für Pathologie, Augsburg, ${ }^{4}$ Klinikum Dortmund, Klinik für Kinderund Jugendmedizin, Dortmund

Kasuistik. Ein 6 Monate alter Säugling wurde mit indolenter Schwellung der Labia majora links vorgestellt. Sonographisch erfolgte der Nachweis eines hernierten und vergrößerten Ovars $(34 \times 14 \mathrm{~mm})$. Im FKDS zeigte sich eine regelrechte Perfusion. Differenzialdiagnostisch wurden ein Ovotestis, Tumor oder DSD erwogen. Intraoperativ imponierte eine große Gonade ohne Anlagepathologie. Dreifache Entnahme eines Feinnadelbiopsates, Belassen der Gonade in situ. Innerhalb von 14 Tagen zeigte sich ein signifikantes Wachstum. Bei histopathologischen Malignitätshinweisen wurde die Indikation zur Adnexektomie links gestellt. Histopathologie. Hochproliferativer juveniler Granulosazelltumor (7-8 Mitosen/1 HPF) mit Nachweis von Inhibin, CD 99. 
Diskussion. Der Tumor wurde als FIGO Ic eingeordnet. Als prognosegünstig wurden das niedrige Alter sowie die Stanzbiospie (im Gegensatz zur intraoperativen Tumorruptur) gewertet. Nach kritischer interdisziplinärer Diskussion präferieren wir eine „Watch-and-waitStrategie" unter radiologischer Kontrolle. Aus chirurgischer Sicht werten wir sowohl die initiale Entnahme von Biopsaten, die konsequente sonographische Verlaufskontrolle vor definitivem histopathologischem Ergebnis wie auch das zweizeitige chirurgische Vorgehen als gerechtfertigt und prognosegünstig.

\section{DGKCH-PO-KC-5 \\ Die intrauterine Katasthrophe: Vanishing bowl bei Laparoschisis - ein Fallbericht}

\section{Cremer M.', Nöth N.', Brückmann D. ${ }^{2}$, Großer K.'}

${ }^{1} K$ linik für Kinderchirurgie und Kinderurologie, HELIOS Klinikum Erfurt, Erfurt, Deutschland, ${ }^{2}$ Praxis für Pränataldiagnostik, Frauenheilkunde und Geburtshilfe, Erfurt, Deutschland

Geburt eines $3120 \mathrm{~g}$ schweren Mädchens per primärer Sectio am 13.11.2012. Pränatal bekannte Gastroschisis, die sich zurückgebildet hat und nach der 20. SSW sonographisch nicht mehr nachweisbar war. Kein Bauchwanddefekt zur Geburt. Signifikanter Untergang des Darmes mit daraus reultierendem kongenitalem Kurzdarm nach fetaler Gastroschisis. Ileumatresie. Dünndarm-Ileus postnatal. Keine weiteren Fehlbildungen. Komplizierter Verlauf mit mehrfachen Laparotomien unter parenteraler Ernährung per Hickman-Katheter. Nach teilweise Enteralisierung Darmverlängerungsoperation extra muros. Im Verlauf dann extra muros an einer Kathetersepsis 12/2013 verstorben.

\section{DGKCH-PO-KC-6}

Ein myoepitheliales Hamartom als seltene Ursache einer ileocolischen Invagination im Kleinkindalter

\author{
Breuling K.', Krohn C. ', Peschke P.', Hosie S.' \\ ${ }^{1}$ Klinikum München Schwabing, Klinik für Kinderchirurgie, München, ${ }^{2}$ Klini- \\ kum München Schwabing, Institut für Pathologie, München
}

Hintergrund. Die Invagination ist eine häufige Ursache eines akuten Abdomens im Kindesalter. Meist finden sich ileocolische Invaginationen. Betroffen sind vor allem Säuglinge und Kleinkinder mit einem Häufigkeitsgipfel im 9. bis 10. Lebensmonat, hierbei handelt es sich meist um idiopathische Invaginationen. Außerhalb der typischen Altersgruppe finden sich häufiger pathologische Leitstrukturen als Ursache.

Kasuistik. Wir berichten von einem 6 Monate alten männlichen Säugling der mit Erbrechen, Trinkschwäche und Fieber in unserer Notaufnahme vorgestellt wurde. Bei blutig-schleimigen Stühlen, starken Bauchschmerzen und gespanntem Abdomen erfolgte eine Sonographie, hierbei zeigte sich eine ileocolische Invagination. Eine hydrostatische Desinvagination war nicht möglich. Es erfolgte die offene Desinvagination. Hierbei mussten ca. $10 \mathrm{~cm}$ nekrotischen Ileums entfernt werden. Als Leitstruktur fiel eine derbe Raumforderung von ca. 1,5 cm Durchmesser auf. Histopathologisch wurde ein myoepitheliales Hamartom nachgewiesen. Der postoperative Verlauf war unauffällig.

Schlussfolgerung. Hamartome als Ursache für Invaginationen im Kindesalter sind bisher nach unserer Kenntnis nur in 8 Fällen beschrieben. Hierbei handelt es sich in 6 Fällen um Dünndarminvaginationen. Über ileocolische Invaginationen liegen bisher nur 2 Berichte von einem 13 und einem 2 Jahre alten Knaben vor. Unser Bericht ist somit der dritte einer ileocolischen Invagination aufgrund eines Hamartoms im Kindesalter und der zweite bei einem Kleinkind. Intestinale Hamartome kommen im Rahmen des Peutz-Jeghers-Syndroms vor. Eine genetische Diagnosestellung ist möglich und sinnvoll, da ca. 50\% der Patienten mit Peutz-Jeghers-Syndrom im Erwachsenenalter Malignome entwickeln und deshalb spezifischer Vorsorge bedürfen.

\section{DGKCH-PO-KC-7}

Vom unklaren Hodentumor zur seltenen Fehlbildung einer splenogonadalen Fusion mit Peromelie und Mikrogenie

\section{Alhussami I.', Heinick C. ${ }^{1}$, Stenzel M. ${ }^{2}$, Nele H. ${ }^{3}$, Eckoldt F. ${ }^{1}$}

'Universitätsklinik für Kinderchirurgie, Jena, ${ }^{2}$ Institut für Diagnostische und Interventionelle Radiologie, Sektion Kinderradiologie, Jena, ${ }^{3}$ Universität Jena, Institut der Humangenetik, Jena

Fallbericht. Wir berichten über eine pränatal nicht bekannte schwere Fehlbildung mit Peromelie der oberen Extremitäten und des linken Beines, Amelie des rechten Beines, mit Aplasie des Os ischium rechts. Vergesellschaftet mit einer Spalte des weichen Gaumens, Mikro- und Retrognathie, Mikropenis mit glandulärer Hypospadie und Splengonadale Fusion.

Methoden. Im Alter von 6 Monaten erfolgte auswärtig eine inguinale Freilegung bei Verdacht auf eine inkarzierierte Leistenhernie. Hier zeigte sich intraoperativ ein unklares längliches Tumorsubstrat, welches vom linken Hoden nach intraabdominell durch den Leistenkanal zog.

Ergebnisse. Die folgende klinische, humangenetische und kinderradiologische Diagnostik im unserem Zentrum ergab den Verdacht auf die selten auftretende kontinuierliche splenogonadale Fusion, welche in einer zweiten Sitzung in toto unter kompletter Erhaltung des Hodens entfernt und histologisch gesichert wurde.

Schlussfolgerung. In der internationalen Literatur sind bisher ca. 150 Fälle bekannt. Der jüngste Patient war ein Neugeborenes, der älteste ein 69-jähriger Mann. Etwa 75\% der Patienten sind bei Diagnosestellung jünger als 20 Jahre. Bisher sind erst deutlich weniger Fälle von splenogonadaler Fusion beim weiblichen Geschlecht bekannt. Bei allen Patienten fanden sich gehäuft verschiedene Formen der Peromelie oder eine Mikroganathie. Das klinische Erscheinungsbild der Splenogonadalen Fusion ist variantenreich. Bei ca. 50\% der Fälle wurde ein linksseitiger Hodentumor als häufigster Befund beschrieben. Auch in unserem Fall erfolgte die Erstvorstellung des Kindes in unserer Klinik mit dem Verdacht auf unklaren Hodentumor.

\section{DGKCH-PO-KC-8}

Gardner-Fibrom eines Neugeborenen - Konsequenzen für die ganze Familie

Schäfer M. ${ }^{1}$, Schmidt W. ${ }^{2}$, Kadmon M. ${ }^{3}$, Sutter C. ${ }^{4}$, Moog U. ${ }^{4}$, Kaiser A. ${ }^{5}$, Stehr $M \cdot{ }^{1}$

'Cnopf'sche Kinderklinik, Abteilung für Kinderchirurgie und Kinderurologie, Nürnberg, ${ }^{2}$ Cnopf'sche Kinderklinik, Abteilung für Kinderheilkunde/ Pädiatrische Hämatologie und Onkologie, Nürnberg, ${ }^{3}$ Universitätsklinikum Heidelberg, Klinik für Viszeralchirurgie, Heidelberg, ${ }^{4}$ Universitätsklinikum Heidelberg, Institut für Humangenetik, Heidelberg, ${ }^{5}$ Klinikum Nürnberg Nord, Institut für Pathologie, Nürnberg

Fragestellung. Die familäre adenomatöse Polyposis (FAP) ist eine seltene Ursache für das kolorektale Karzinom und wird durch ein weites Spektrum von Mutationen im APC-Gen verursacht. Das Auftreten von Garder-Fibromen, einer erst in den letzten Jahren beschriebenen, sehr seltenen Tumorentität wurde mit dem Vorhandensein einer FAP assoziiert.

Material und Methoden. Ein 7 Wochen alter männlicher Säugling wurde mit einer seit Geburt bestehenden derben Schwellung der linken Brustwand vorgestellt. Sonographisch zeigte sich ein schlecht abgrenzbarer $1,48 \times 0,48 \mathrm{~cm}$ großer Tumor. Es wurde eine subtotale Exzision durchgeführt. Histologisch bestätigte sich der Verdacht auf ein Gardner-Fibrom. In den nächsten Monaten kam es erneut zu einem Wachstum des Tumors mit Infiltration der Rippen, so dass im Alter von neun Monaten eine totale Resektion mit Rippenteilresektion und Entfernung des distalen Sternums durchgeführt. Der Brustwandverschluss gelang mit Einlage eines Goretex-Patches sowie eines Pectoralisschwenklappens. 
Ergebnisse. Die histologische Untersuchung bestätigte die Ro-Resektion. Der postoperative Verlauf war unauffällig. Ein Jahr postoperativ besteht im MRT weiterhin kein Hinweis auf Rezidiv. Aufgrund des Gardner-Fibroms wurde eine genetische Untersuchung des Patienten und der Eltern durchgeführt, die zur Entdeckung einer bislang unbekannten APC-Mutation (und somit einer FAP) beim Patienten und seinem Vater führte. Aufgrund dieses Befundes musste beim Vater eine vollständige Kolektomie bei multiplen Adenomen durchgeführt werden.

Diskussion. Die Diagnose einer FAP beim Vater des Patienten stellt ein bislang unbeschriebenes Novum dar und betont, dass das Auftreten eines Gardner-Fibroms im Kindesalter zwingend eine Diagnostik hinsichtlich des Vorliegens einer FAP erfordert.

\section{DGKCH-PO-KC-9}

Reifes Teratom des Mediastinums bei einem 10-monatigen Kleinkind als Ursache für Gedeihstörung

\section{Schäfer M.', Schmidt W. ${ }^{2}$, Mika T. ${ }^{1}$, Kaiser A. ${ }^{3}$, Stehr M. ${ }^{\text {' }}$}

${ }^{1} \mathrm{Cnopf}$ 'sche Kinderklinik, Abteilung für Kinderchirurgie und Kinderurologie, Nürnberg, ${ }^{2}$ Cnopf'sche Kinderklinik, Abteilung für Kinderheilkunde/ Pädiatrische Hämatologie und Onkologie, Nürnberg, ${ }^{3}$ Klinikum Nürnberg Nord, Institut für Pathologie, Nürnberg

Fragestellung. Mediastinale Teratome gehören zu den seltenen gutartigen Tumoren. Über $40 \%$ aller Teratome entstehen im Sakralbereich, sowie weniger häufig im Ovar (25\%) und anderen Lokalitäten. Die Literatur zu dieser Entität beschränkt sich auf wenige Fallberichte und kleine Serien.

Material und Methoden. Ein 10-monatiges weibliches Kleinkind wurde in der Ambulanz vorgestellt wegen rezidivierendem Erbrechen und Gedeihstörung seit vier Monaten. Das Gewicht bei Aufnahme betrug $6640 \mathrm{~g}$ (<3. Perz.). Bei auskultatorisch fehlendem Atemgeräusch auf der linken Lunge wurde ein Röntgenthorax angefertigt, der eine vollständige Verschattung der linken Lunge zeigte. In der daraufhin durchgeführten MRT-Bildgebung zeigte sich ein riesiger Tumor des gesamten linken Hemithorax mit Mediastinalshift nach rechts und Kompression der linken Lunge. Aufgrund der klinischen Symptomatik und des Verdachtes auf ein Teratom wurde eine mediane Sternotomie durchgeführt und der Tumor in toto reseziert.

Ergebnisse. Intraoperativ zeigte sich eine parenchymatöse Brücke zum kaudalen Rand des Thymus. Histologisch bestätigte sich der Verdacht auf ein reifes Teratom ohne Malignität. Postoperativ zeigten sich eine rasche Entfaltung der Lunge sowie Sistieren des Erbrechens und eine rasche Gewichtszunahme.

Diskussion. Unspezifische Allgemeinsymptome wie Erbrechen oder Gedeihstörung waren bei diesem Kind der einzige primäre Hinweis auf eine außergewöhnliche Tumorentität. Dies betont die Notwendigkeit einer ausführlichen körperlichen Untersuchung, um auch seltene Tumorentitäten wie mediastinale Teratome frühzeitig zu entdecken - dies umso mehr, da die chirurgische Resektion in toto die einzige Therapieoption darstellt und bei Tumoren dieser Größe eine Herausforderung darstellen kann

\section{DGKCH-PO-KC-10}

\section{Akute Bauchschmerzen als Erstmanifestationssymptom einer supraventrikulären Tachykardie bei einem 3-jährigen Mädchen - ein Fallbericht}

\section{Eckert K. ${ }^{1}$, Weyers B. ${ }^{1}$, Bienemann $K_{.}{ }^{2}$, Hagemann . $^{2}$, Liedgens $P .{ }^{1}$}

${ }^{1}$ Elisabeth-Krankenhaus Essen, Klinik für Kinderchirurgie, Essen, ${ }^{2}$ ElisabethKrankenhaus Essen, Klinik für Kinder- und Jugendmedizin, Essen

Einführung. Akute Bauchschmerzen sind ein häufiger Vorstellungsgrund in kinderchirurgischen Notaufnahmen und zeigen ein breites differenzialdiagnostisches Spektrum. Eine supraventrikuläre Tachy- kardie (SVT) kann infolge eines Rechtsherzversagens auch starke Bauchschmerzen verursachen. Sie ist aber in der täglichen Praxis eine seltene Differentialdiagnose, so dass diese Patienten, insbesondere für den erstuntersuchenden Kinderchirurgen, eine diagnostische Herausforderung sein können.

Fallbericht. Ein 3-jähriges, bislang gesundes Mädchen wurde mit progredienten kolikartigen Bauchschmerzen kinderchirurgisch vorgestellt. Wegen der starken Schmerzen war eine adäquate Untersuchung erst nach Analgesie mit Piritramid möglich. Danach zeigte sich ein unauffälliger klinisch-körperlicher Untersuchungsbefund. Sonographisch fielen ein Gallenblasenwandödem, echofreier Aszites, eine periportale Echogenitätssteigerung, sowie eine Lebervenendilatation auf. Für andere, v. a. kinderchirurgisch relevante, Bauchschmerzursachen ergab sich kein Anhalt. Bei einem RR von 88/6o mmHg fiel aber eine Tachykardie von 250-260/min auf, die sich im EKG als SVT mit schmalen Kammerkomplexen erwies. Nach pädiatrischer Übernahme der Pat. gelang erst mit 2 mg Adenosin eine Konversion in den Sinusrhythmus. Die üblichen Routinelaborwerte waren nun einsehbar und unauffällig. Das nachgeforderte proBNP war mit $8028 \mathrm{pg} / \mathrm{ml}$ stark erhöht.

Schlussfolgerung. Die Bauchschmerzen und die sonographischen Befunde sind am ehesten im Sinne einer Stauungshepatocholezystopathie als Folge einer SVT-bedingten Rechtsherzinsuffizienz zu erklären. Wie unser Fall zeigt, kann sich eine SVT auch mit akuten Bauchschmerzen manifestieren. Daher sind in der Abklärung akuter Bauchschmerzen immer auch extraabdominale Ursachen zu berücksichtigen.

\section{Posterbegehung 2}

\section{DGKCH-PO-CH-1}

\section{Operative Behandlungsergebnisse suprakondylärer Oberarm-} brüche beim Kind

Ulmar B. ', Schwarz J. ${ }^{2}$, Stöckle U. ${ }^{2}$, Mittlmeier T. , Freude T. ${ }^{2}$

${ }^{1}$ Abt. Unfall-, Hand- und Wiederherstellungschirurgie, Chirurgische Klinik und Poliklinik, Universitätsmedizin Rostock, Rostock, ${ }^{2}$ Klinik für Unfall- und Wiederherstellungschirurgie, BG Unfallklinik Tübingen, Tübingen

Fragestellung. Wie sind die Ergebnisse nach gekreuzter KirschnerDraht-Osteosynthese bei suprakondylären Oberarmbrüchen im eigenen Patientengut?

Methoden. Kinder mit suprakondylären Oberarmbrüchen aus den Jahren 2000-2008 wurden retrospektiv auf frakturspezifische und epidemiologische Parameter hin analysiert und postoperativ bezüglich ihrer Funktion und Zufriedenheit mit einem individuellen Fragebogen und verschiedenen Scores untersucht.

Ergebnisse. 46 Patienten (Median: 7 Jahre) wurden eingeschlossen. Jungen $(63 \%)$ und die linke Seite $(54 \%)$ waren bevorzugt betroffen. Offene Frakturen ( $2 \%$ ) oder Gefäß- (o\%) und Nervenschäden (4\%) waren selten, begleitende Unterarmfrakturen (15\%) häufig. Die Versorgung erfolgte durch Reposition und gekreuzte Kirschner-Drähte. Hauptkomplikationen waren Bewegungseinschränkungen und Draht-Migrationen. Alle Frakturen durchbauten knöchern. $72 \%$ der Patienten wurden durchschnittlich 51 Monate postoperativ nachuntersucht: Die Differenz zwischen nicht betroffenem und betroffenem Arm betrug im Schnitt in Flexion $8^{\circ}$, in Extension $1^{\circ}$, in Pronation $1^{\circ}$ und in Supination $0^{\circ}$. Die Unterschiede für den Valgus-Winkel und die Armlänge zwischen nicht betroffenem und betroffenem Ellenbogen betrugen durchschnittlich $0^{\circ}$. Im MEPS erreichten 88\% der Patienten exzellente oder gute Ergebnisse. Schmerzen bestanden bei $15 \%$ der Patienten bei einem Schmerzausmaß von durchschnittlich 2 Punkten auf der VAS.

Schlussfolgerung. Offene Frakturen oder Gefäß- und Nervenschäden sind selten, simultane Unterarmfrakturen häufig. Aufgrund der Frakturheilung in allen Fällen, gut zu beherrschenden Komplikationen, guten bis exzellenten Werten im MEPS und guter Funktion, ist die gekreuzte Kirschner-Draht-Osteosynthese nach Reposition ein sicheres 
Standardverfahren für die Versorgung von suprakondylären Oberarmbrüchen beim Kind.

\section{DGKCH-PO-CH-2}

Therapie kindlicher Femurfrakturen: 2 versus 3 elastischen stabilen intramedullären Nägeln

\author{
Semaan A. ${ }^{1}$, Boemers T. ${ }^{1}$ \\ ${ }^{1}$ Kliniken der Stadt Köln gGmbH, Klinik für Kinderchirurgie und Kinderuro- \\ logie, Kinderkrankenhaus Amsterdamer Straße, Köln
}

Fragestellung. Die konservative Behandlung kindlicher Femurfrakturen kann aufgrund der langen Immobilisation die Patienten und deren Familie sozial und finanziell belasten. Aufgrund einer kürzeren Immobilisation haben operative Therapieansätze immer mehr an Bedeutung bei der Versorgung dieser Frakturen gewonnen. In dieser Studie soll die Stabilisierung von Oberschenkelfrakturen durch 3 gegen 2 ESIN verglichen werden. Unsere Hypothese ist, dass das Einbringen eines dritten ESIN zu einer besseren Stabilisierung der Femurfraktur und daher zu einem besseren postoperativen Ergebnis führt.

Methodik. Retrospektive Auswertung aller im Hause behandelten Oberschenkelfrakturen vom 2009 bis 2012. Follow-up mit klinischer Untersuchung und standardisierte Erfassung des Bewegungsausmaßes und der Beinlängen im Sommer 2013.

Ergebnisse. Im Follow-up zeigte sich eine signifikante Beinlängendifferenz der durch 3 ESIN versorgten Frakturen $(p=0,013)$, sowie ein signifikanter Unterschied in der Bewegung im Hüftgelenk ( $\mathrm{p}=0,029)$. Zudem war die Operationsdauer bei Metallentfernung in der Gruppe mit 3 ESIN signifikant länger $(p=0,046)$. Alle weiteren untersuchten Parameter zeigten keinen signifikanten Unterschied.

Diskussion. Die Versorgung von Femurschaftfrakturen bei Kindern durch 3 ESIN zeigt in dieser Studienpopulation keinen Vorteil gegenüber einer Versorgung durch 2 ESIN. Die Längendifferenz bei der Versorgung durch 3 ESIN ist möglicherweise durch die nicht symmetrische Anordnung der Abstützungspunkte und eine daraus resultierende vermehrte Mikrobewegung mit erhöhter Kallusbildung erklärbar.

\section{DGKCH-PO-CH-3 \\ Klinische und radiologische Ergebnisse nach Osteosynthese von kindlichen Frakturen des Epikondylus humeri ulnaris}

Ulmar B. ${ }^{1}$, Schwarz J. ${ }^{2}$, Stöckle U. ${ }^{2}$, Mittlmeier T. ${ }^{1}$, Freude T. ${ }^{2}$

${ }^{1}$ Abt. Unfall-, Hand- und Wiederherstellungschirurgie, Chirurgische Klinik und Poliklinik, Universitätsmedizin Rostock, Rostock, ${ }^{2}$ Klinik für Unfall- und Wiederherstellungschirurgie, BG Unfallklinik Tübingen, Tübingen

Fragestellung. Wie sind die Ergebnisse nach Osteosynthese von kindlichen Frakturen des Epicondylus humeri ulnaris im eigenen Krankengut?

Methodik. Aus den Jahren 2000-2008 wurden Kinder mit Osteosynthese einer Fraktur des Epicondylus humeri ulnaris retrospektiv mit ihren frakturspezifischen und epidemiologischen Parameter erfasst. Die Nachuntersuchung analysierte die klinische Funktion und Zufriedenheit mit einem individuellen Fragebogens und verschiedenen Scores.

Ergebnisse. 22 Patienten (Meidan: 11 Jahren) wurden eingeschlossen. $59 \%$ der Patienten waren Jungen, $41 \%$ Mädchen. In 55\% war die rechte Seite betroffen. Zwei Kinder zeigten neurologische Defizite, 7 Kinder eine begleitende Ellenbogengelenksluxation und 1 Kinder eine begleitende proximale Radiusfraktur. Die Operationsdauer betrug im Schnitt 71 Minuten. Zur operativen Stabilisierung wurden Schrauben, Kirschner-Drähte oder die Kombination aus beidem eingebracht. Drei Patienten hatten postoperative Komplikationen. Eine knöcherne Durchbauung zeigte sich in allen Fällen. 91\% unserer Patienten wurden durchschnittlich 4 Jahre postoperativ nachuntersucht. Der betroffene Ellenbogen hatte folgende durchschnittliche Bewegungsausmaße: Flexion $140^{\circ}$, Extension $3^{\circ}$, Pronation $86^{\circ}$ Supination $89^{\circ}$. Der VAS-Score zeigte durchschnittlich 2 Punkte. $85 \%$ der Patienten zeigten im MEPS exzellente oder gute, $15 \%$ zufriedenstellende Resultate. Ein Patient hatte anhaltende neurologische Defizite.

Schlussfolgerung. Eine Kombination mit Ellenbogenluxationen ist häufig, weitere Frakturen oder Nerven-/Gefäßschäden hingegen selten. Nach Osteosynthese zeigen sich gute subjektive Ergebnisse und eine gute objektive Funktion mit moderaten Einschränkungen. Vor diesem Hintergrund und angesichts der nach Literatur geringeren Pseudarthrosen-Rate, sollte die Indikation zur operativen Stabilisierung großzügig gestellt werden.

\section{DGKCH-PO-CH-4}

Superantigen-Arthritis - ein neues Tiermodell für die juvenile idiopathische Arthritis (JIA)?

Gerlach K. ${ }^{1}$, Tomuschat C. ', Staege M. ${ }^{2}$, Brandt J. ${ }^{3}$, Finke R. ${ }^{1}$, Kornhuber M. ${ }^{4}$, Emmer $A .{ }^{4},{ }^{2}$

'Martin-Luther-Universität Halle-Wittenberg, Klinik und Poliklinik für Kinderchirurgie, Halle/Saale, ${ }^{2}$ Martin-Luther-Universität Halle-Wittenberg, Klinik und Poliklinik für Kinderheilkunde und Jugendmedizin, Halle/Saale, ${ }^{3}$ Martin-Luther-Universität Halle-Wittenberg, Klinik und Poliklinik für Orthopädie, Halle/Saale, ${ }^{4}$ Martin-Luther-Universität Halle-Wittenberg, Klinik und Poliklinik für Neurologie, Halle/Saale

Fragestellung. Die Autoimmunarthritis (AA; z. B. die JIA) ist eine chronisch inflammatorische Erkrankung des Synovialgewebes, die zur Gelenkdestruktion führt. Die Ätiologie ist weitgehend unbekannt. Verschiedene Bakterien und Viren sowie deren Superantigene werden mit der Pathogenese in Verbindung gebracht. Aus diesem Grund untersuchten wir das arthritogene Potential des bakteriellen Superantigens Staphylokokken Enterotoxin A (SEA).

Material und Methoden. $50 \mu \mathrm{l} \mathrm{SEA}(1 \mathrm{mg} / \mathrm{ml})$ wurden in das rechte Kniegelenk von 9 männlichen, $300 \mathrm{~g}$ schweren Lewis-Ratten während tiefer Isoflurannarkose injiziert. Neun Tiere erhielten $50 \mu \mathrm{NaCl}$ o,9\% intraartikulär. 1, 5 und 10 Tage nach intraartikulärer Injektion von SEA bzw. $\mathrm{NaCl}$ o,9\% wurden jeweils 6 Tiere geopfert, die Kniegelenke entnommen, entkalkt und Mikrotomschnitte angefertigt. Histologische (HE) sowie immunhistochemische Färbungen gegen die Antigene $\mathrm{CD}_{3}$, CD4, CD8, CD138, Pax-5, MHC-I und MHC-II wurden durchgeführt. Ergebnisse. Nach intraartikulärer Injektion fand sich eine prominente Rundzellinfiltration subsynovial, insbesondere im oberen Rezessus sowie an der Basis des lateralen und medialen Meniskus. Fünf Tage nach intraartikulärer Injektion wurde die maximale Entzündungsaktivität detektiert. Die Anzahl der CD8+ T-Zellen lag stets über der Summe aus $\mathrm{CD}_{3}+$ und $\mathrm{CD}_{4}+\mathrm{T}-Z$ ellen. Die Expression von MHC-I+und MHC-II+ Zellen stieg im Verlauf der Beobachtungszeitpunkte. Nur wenige Plasmazellen (CD138+) und B-Zellen (Pax-5+) waren nachweisbar. In den Gelenken der Kontrolltiere fand sich durchweg eine nur marginale signifikant geringere Rundzellinfiltration.

Diskussion. Wir konnten nachweisen, dass die intraartikuläre Expression von SEA in der Lewis-Ratte zu einer Arthritis führt, die deutliche Ähnlichkeiten im Entzündungsbild zur JIA aufweist.

\section{DGKCH-PO-CH-5}

Pleuraempyem: Behandlungsstrategie am Otto-Heubner-Zentrum der Charité Berlin

\section{Schwarz B. ${ }^{1}$, Rothe K. ${ }^{1}$, Heimann A. ${ }^{1}$, Mehl A. ${ }^{2}$}

'Charité, Universitätsmedizin Berlin, Klinik und Poliklinik für Kinderchirurgie, Berlin, ${ }^{2}$ Klinik für Kinderpulmologie, Charité, Berlin

Fragestellung. Trotz Impfung und verbesserter infektiologischer Behandlung steigt die Inzidenz von Pleuraempyemen bei Kindern in den letzten Jahren. Die Behandlungskonzepte reichen von primär konservativen Verfahren wie Punktion mit Instillation von Urokinase, thorakoskopischer Platzierung von Drainagen (VATS) bis zur Thorakotomie 
und Dekortikation. Es besteht kein Konsens bezüglich der optimalen Vorgehensweise.

Material und Methode. Analyse der Kinder mit Pleuraerguss/Pleuraempyem am Otto-Heubner-Zentrum der Charité, Universitätsmedizin Berlin, die interdisziplinär in den letzten 6 Jahren behandelt wurden. Hierbei wird das Vorgehen, beginnend mit den diagnostischen Maßnahmen über das entsprechende therapeutische Verfahren, die Behandlungsdauer, Komplikationen und deren Management dargestellt. Diskussion. Das eigene Behandlungskonzept folgt einem interdisziplinären diagnostischen und therapeutischen Algorithmus, der in zäher Auseinandersetzung, aber ergebnisorientiert erarbeitet wurde. Es wird besonders auf die enge Zusammenarbeit mit den Kinderpulmologen eingegangen.

\section{DGKCH-PO-CH-6}

Chirurgische Therapie des fokalen kongenitalen Hyperinsulinismus - eine interdisziplinäre Herausforderung

\section{Varol E. , Mohnike K. ${ }^{2}$, Müller C. ${ }^{1}$, Vogelgesang S. ${ }^{3}$, Schröder C. ${ }^{4}$, Mohnike W. ${ }^{5}$, Wieland $1 .{ }^{6}$, Zenker M. ${ }^{6}$, Barthlen W. \\ 'Universitätsmedizin Greifswald, Kinderchirurgie, Greifswald, ${ }^{2}$ Universitäts- klinik Magdeburg, Kinderendokrinologie, Magdeburg, ${ }^{3}$ Universitätsme- dizin Greifswald, Institut für Pathologie, Greifswald, ${ }^{4}$ Universitätsmedizin Greifswald, Pädiatrie, Greifswald, ${ }^{5}$ Diagnostisch Therapeutisches Zentrum Frankfurter Tor, Berlin, 'Universitätsklinik Magdeburg, Humangenetik, Magdeburg}

Einleitung. Die fokale Form des kongenitalen Hyperinsulinismus ist durch eine selektive Resektion heilbar. Präoperativ ist die molekulargenetische Diagnostik des Patienten, der Patienteneltern sowie die exakte Lokalisation mittels 18F DOPA-PET/CT unverzichtbar. Während der Operation besteht ein enger Informationsaustausch mit der Pathologie durch multiple Gewebebiopsien.

Patienten und Methoden. In den Jahren 12/2009 bis 12/2013 wurden 22 Kinder zwischen dem 2. Lebensmonat und dem 3. Lebensjahr mit einem fokalen kongenitalen Hyperinsulinismus in unserer Klinik operiert. Ergebnisse. Alle Kinder wiesen eine paternal homozygote Mutation auf, darunter 17 Kinder im ABCC8 Gen und fünf im KCNJ11 Gen. Es bestand keine Korrelation zwischen der genetischen Konstellation und der Lokalisation des Fokus. Von den 22 Patienten wurden 10 minimal-invasiv operiert und 12 offen chirurgisch. Bei sieben Patienten wurde eine Roux-en-Y-Anastomose durchgeführt. An Komplikationen erlitt ein Kind mit Faktor V Leiden Mutation intraoperativ eine Lungenembolie, ein weiteres Kind bot drei Monate nach Operation einen Adhäsionsileus und ein Kind wurde 5 Tage nach Operation bei nekrotisierender Enterokolitis erneut operiert. Ein Kind wurde nur biopsiert Ein Kind wurde zweimal operiert, eines dreimal. 20 von 21 Kindern mit kurativer Intention konnten geheilt werden (95\%). Das mittlere Followup beträgt 18,5 Monate.

Schlussfolgerung. Die interdisziplinäre Zusammenarbeit ist für den Therapieerfolg beim kongenitalen Hyperinsulinismus essentiell. Die limitierte Enukleation hat eine Erfolgsrate von 95\%.

\section{DGKCH-PO-CH-7}

Therapie eines 10 Jahre alten Jungen mit operativer Sanierung einer Echinokokkuszyste in der Lunge ohne komplette Perizystektomie

\section{Hoffmann S.', Stoelben E. ${ }^{2}$, Kellner M.W. ${ }^{3}$, Boemers T.M.}

${ }^{1}$ Kinderkrankenhaus der Kliniken der Stadt Köln, Kinderchirurgie und Kinderurologie, Köln, ${ }^{2}$ Lungenklinik der Kliniken der Stadt Köln, Thoraxchirurgie, Köln, ${ }^{3}$ Kinderkrankenhaus der Kliniken der Stadt Köln, Kinderradiologie, Köln

Material und Methoden. Es handelt sich bei dem Patienten um einen 10 Jahre alten Jungen aus Ägypten, der seit 2 Monaten in Deutschland ansässig ist und klinisch mit rezidivierenden Atemwegsinfekten und Hämoptysen auffällig wurde. Die Einweisung erfolgte zum Ausschluss einer Tuberkulose. Ein Röntgen-Thorax zeigte pulmonale Infiltrate beidseits. Im MRT des Thorax zeigte sich der dringende V. a. eine Echinokokkuszyste beidseits. In der Serologie konnten Antikörper gegen Echinococcus granulosus nachgewiesen werden. Aufgrund der Größe und des Anschlusses an das Bronchialsystem wurde die Indikation zur operativen Sanierung der linksseitigen Zyste gestellt und eine perioperative Behandlung mit Albendazol begonnen.

Ergebnisse. Über eine laterale Thorakotomie links wurde der Zysteninhalt abgesaugt, ohne den Pleuraraum zu kontaminieren, die Perizyste wurde teilweise exzidiert und 3 Bronchusfisteln verschlossen. Danach erfolgte die Spülung der Zystenhöhle mit $\mathrm{NaCl} 20 \%$ und im Anschluss mit $\mathrm{NaCl}$ o,9\%. Eine eingelegte Thoraxdrainage wurde für 5 Tage postoperativ belassen. Insgesamt zeigte sich ein komplikationsloser postoperativer Verlauf. Es erfolgte eine postoperative Behandlung mit Albendazol für 3 Monate. Die Verlaufskontrolle ist für Juni 2014 geplant. Diskussion. Die angewandte operative und medikamentöse Therapieoption soll anhand des Outcomes im Vergleich zur Studienlage diskutiert werden.

\section{DGKCH-PO-CH-8}

Hydrometrokolpos bei persistierendem Sinus urogenitalis mit progressiver Harnstauung einer Einzelniere - ein seltener kinderchirurgischer Notfall in der Neonatalperiode

Kretschmer K.', Girschick H.J.', Schunck K.-U.', Tillig B. ${ }^{2}$

'Vivantes Klinikum im Friedrichshain, Klinik für Kinder-und Jugendmedizin/ Perinatalzentrum, Berlin, ${ }^{2}$ Vivantes Klinikum Neukölln, Vivantes Klinikum im Friedrichshain, Kinder-und Neugeborenenchirurgie/ Kinderurologie, Berlin

Hintergrund. Ein konnataler Hydrometrokolpos ist eine ausgesprochene Rarität und kann sich, neben der diagnostischen Herausforderung, zu einem kinderchirurgischen Notfall entwickeln.

Fallbericht. Wir berichten über ein reifgeborenes Mädchen, welches mit einer massiven abdominellen Raumforderung vorgestellt wurde. Pränatal wurden eine singuläre Umbilikalarterie (SUA), eine Nierenagenesie rechts und der V. a. eine Ovarialzyste diagnostiziert. Die klinische Untersuchung war regelrecht, das äußere Genital zeigte jedoch keinen Introitus vaginae und nur eine Öffnung, aus der sich Urin entleerte. Ergebnisse. Die weitere Bildgebung (Sono, MRT) ergab einen $8 \times 6 \times 4 \mathrm{~cm}$ großen flüssigkeitsgefüllten Tumor im kleinen Becken, dessen Ursprung nicht eindeutig identifizierbar war. Zusätzlich zeigte sich eine Doppelniere links mit progressiver Hydronephrose III'. Am 12. LT wurde laparoskopisch eine Pigtail-Drainage in die Zyste eingebracht, aus der sich $100 \mathrm{ml}$ trübes Transsudat entleerte. Die beschriebene Harnstauung bildete sich zurück. Topografisch konnte die zystische Struktur a. e. dem Uterus zugeordnet werden, eine Müllergang-Anomalie war nun naheliegend. Die drei Monate später erfolgte Endoskopie ergab einen persistierenden Sinus urogenitalis mit einer durch ein Septum verschlossenen Mündung der Anlagen von Vagina und Uterus. Zystoskopisch identifizierten wir zudem eine Ureterozele links in Blasenhalshöhe, welche gefenstert wurde.

Schlussfolgerung. Unserer Kenntnis nach, wurde solch ein Fall (Hydrometrokolpos, Sinus urogenitalis, Doppelniere, Ureterozele, kontralat. Nierenagenesie, SUA) bisher nicht publiziert. Er beweist einmal mehr, dass bei Mädchen mit Nierenfehlbildungen das innere Genital genauestens inspiziert werden sollte und dass bei sekundären Harntransportstörungen operative Interventionen bereits in der Neonatalzeit erforderlich sein können. 


\section{DGKCH-PO-CH-9}

Plötzlicher Herztod und Empfehlungen zur Prävention der Commotio cordis

\section{Eyermann $R^{1}$}

'Dr. Richard Eyermann, Kinder- und Jugendmedizin, Kinderkardiologie, Sportmedizin, München

Problemstellung. PHT ist ein zwar seltenes aber dramatisches Ereignis. Fragestellung. Wie kann dem PHT, v. a. durch Commotio-cordisEvents, vorgebeugt werden?

Methodik. Literaturrecherche und Erarbeitung evidenzbasierter Empfehlungen.

Ergebnisse. Definition: Commotio cordis ist definiert als VF und PHT, ausgelöst durch einen stumpfen, nicht penetrierenden Schlag gegen den Thorax, ohne Schäden an Rippen, Sternum oder Cor - und dies ohne kardiovaskuläre Grunderkrankung.

Differenzialdiagnose. HOCM, LQTS, Brugada-Syndrom, Mi in der Kindheit, ARVD, Virusmyokarditis, ALCAPA, Elektrolytanomalien. Epidemiologie. Über 224 Fälle in den USA seit Einführung der Commotio-cordis-Registry 1995 gemeldet, große Dunkelziffer. Events-Betroffene: Überlebensrate $24 \%$ ! 95\% männlichen Geschlechts. Inzidenzgipfel 10-18 Jahre. 50\% Ereignisse im Leistungssport, 25\% im Freizeitsport, $25 \%$ übrige, z. B. Tritt vom Pferd. Baseball hat höchste Inzidenz, dann Softball, Hockey, Football.

Diskussion und Schlussfolgerung. Präventionsansätze: Öffentlicher Zugang zu Defibrillatoren bei Sportveranstaltungen mit hoher Inzidenz von Commotio-cordis-Ereignissen und Ausbildung von Menschen in Anwendung von Defis und kardiopulmonaler Reanimation (CPR) ist wirksamste Maßnahme um das Überleben von Menschen, die PHT erleiden, zu verbessern. Sinnvoll ist weiter öffentliche Aufklärung über die Verhütung präkordialer Gewalteinwirkung, z. B. sollten Baseball- und Softball-Trainer ermuntert werden die Spieler zu erziehen, dem Schlag bzw. der traumatischen Ballwirkung rechtzeitig auszuweichen oder die Bälle mit der rechten Schulter anstelle der Brustwand abzuwehren (Ausweichtraining). Thoraxschilde sind weitgehend unwirksam. 18\% der Commotio-cordis-Fälle trugen ein Brustschild. Intensivere Polsterung schränkt sportartspezifische Leistungsfähigkeit zu sehr ein. 